\title{
CILIATES ON THE MACROPHYTES IN INDUSTRIALLY HEATED LAKES (KUJAWY LAKELAND, POLAND)
}

\author{
R. Babko ${ }^{1}$, J. Fyda ${ }^{2}$, T. Kuzmina ${ }^{3}$, A. Hutorowicz ${ }^{4}$ \\ ${ }^{1}$ Sumy State Pedagogical University, \\ Romens'ka str. , 87, Sumy, 40002 Ukraine \\ E-mail: rbabko@ukr.net \\ 2 Institute of Environmental Sciences, Jagiellonian University, \\ Gronostajowa str., 7, Krakow, 30-387 Poland \\ E-mail: janusz.fyda@uj.edu.pl \\ ${ }^{3}$ Sumy State University, Rimskogo-Korsakova str., 2, Sumy, 40007 Ukraine \\ E-mail: kuzmina_tm@ukr.net \\ ${ }^{4}$ Inland Fisheries Institute in Olsztyn, 10, Olsztyn-Kortowo, 10-719 Poland \\ E-mail:ahut@infish.com.pl
}

Received 30 October 2009

Accepted 26 July 2010

Ciliates on the Macrophytes in Industrially Heated Lakes (Kujawy Lakeland, Poland). Babko R., Fyda J., Kuzmina T., Hutorowicz A. - The ciliate assemblage on the macrophytes was examined in 2005 during the vegetation period in the Konińskie Lakes which are heating by post-cooling waters from thermal electric plants. As a result of changed temperature regimen the alien thermophilic macrophyte Vallisneria spiralis is becoming increasingly common in the littoral zone. A total of 150 ciliate taxa belonging to 27 orders were found. Greater ciliate species diversity was found on architecturally complex, submerged forms such as Ceratophyllum demersum and Myriophyllum spicatum. By contrast the ciliate compositions on emergent macrophytes with simple architecture in their submerged parts, such as Typha, Sparganium, or Acorus, were less species rich. Despite the simple architecture of Vallisneria leaves, the ciliate diversity on them was high. The results show that replacement of native macrophytes by the alien form $V$. spiralis in heated lakes did not impoverish the ciliate diversity.

Key words: ciliate, macrophytes, diversity, lakes.

Ресничные простейшие на макрофитах в озерах, подверженных тепловому загрязнению (озерный край Куявы, Польша). Бабко Р., Фыда Я., Кузьмина Т., Хуторович А. - В течение вегетационного периода 2005 г. изучали ассамблею ресничных простейших на макрофитах в Конинских озерах, подверженных тепловому загрязнению, которое вызвано поступлением подогретых вод с теплоэлектростанции. Вследствие изменения температурного режима водоемов, в их литорали доминирующее положение среди макрофитов занял адвентивный вид Vallisneria spiralis. Всего на макрофитах было зарегистрировано 150 таксонов ресничных простейших, относящихся к 27 отрядам. Большее разнообразие видов ресничных простейших обнаруживалось на пространственно сложноорганизованных поверхностях таких макрофитов, как Ceratophyllum demersum и Myriophyllum spicatum. На макрофитах с простой архитектурой, таких как, например, Typha, Sparganium и Acorus, количество видов было меньшим. В то же время, несмотря на простую архитектуру листьев Vallisneria, разнообразие ресничных простейших на них было высоким. Таким образом, вытеснение аборигенных макрофитов адвентивным видом $V$. spiralis в условиях подогретых озер не приводило к снижению разнообразия ресничных простейших.

Ключевые слова: ресничные простейшие, макрофиты, разнообразие, озера.

\section{Introduction}

In freshwater lakes, periphyton communities develop mainly in the littoral zone on the submerged surfaces of water plants (Wetzel, 1983). Macrophytes differ strongly in the architecture of leaves and stems, as well as in the texture of plant surfaces and as a natural substrate for different periphytic species with both chemical and physical parameters of lake water, affect periphytic communities structure and species 
composition occurring on them (Raffaelli et al., 2000; Wetzel, 2001). Consequently the number of possible niches offered for a variety of periphytic species is enormous, so differences in species composition and density on different macrophytes are likely to occur (Messyasz, Kuczyńska-Kippen, 2006; Mieczan, 2007; Pals et al., 2006).

Periphytic communities are composed of a range of organisms including ciliated protozoa which are among the least studied, yet play an important role as consumers of bacteria, flagellates, and periphytic algae (Sleigh et al., 1992; Primc-Habdija, Radanowic, 1998). Recent studies have demonstrated that the composition of ciliate periphyton communities in both marine and freshwater habitats is influenced by water chemistry, especially the availability of nutrients (Gong et al., 2005; Mieczan, 2005; Primc-Habdija et al., 2001; Wickham et al., 2004). However, it is uncertain whether it is the physicochemical parameters of the lake or the kinds of macrophyte present as a substrate for attachment that is most important in determining the periphyton species composition (Messyasz, Kuczyńska-Kippen, 2006).

In the lakes investigated during the present study, an increase in the water temperature caused a change in the hydrophyte composition, the most conspicuous being the appearance of an alien thermophile macrophyte Vallisneria spiralis, which appeared in lakes in the mid-1990s (Gabka, 2002; Hutorowicz et al., 2006; Protasov et al., 1994). As V. spiralis becomes more abundant in Konińskie Lakes (Hutorowicz et al., 2006 ) and builds submerged mono-species water meadows, the native submerged macrophytes move to the deeper parts of lake. The effects of this process on the ciliate diversity in periphyton, however, are still unknown. The aim of this study was to determine the ciliate diversity and assemblage composition occurred in heated lakes both on alien ( $V$. spiralis, Eichhornia crassipes (Mart.)) and native macrophytes.

\section{Material and methods}

The studied lakes Slesińskie, Mikorzyńskie, and Licheńskie are situated in Central Poland in the Kujawskie Lakeland. These are typically postglacial, eutrophic lakes suffering from strong anthropopression. The main stressing factor is the introduction of post cooling, heated water from Patnyw-Adamyw-Konin power plants to the lake system. As a result of industrial pressure, higher in comparison to other lakes in the region, there has been an observed increase in the mean water temperature of about $7.5-9.5^{\circ} \mathrm{C}$ (Socha, Zdanowski, 2001). These lakes also have a very short retention times (between 3 and 14 days on average in 1987-2000) and are connected to one another by a series of canals and locks. We chose three sampling stations located in those three lakes of the system. They were situated in shallow gulfs where the abundance and species richness of the macrophytes were both high.

In addition to the typical macrophytes originally found in these lakes and present in similar lakes, the invasion of the alien species Vallisneria spiralis has been observed since 1990 (Protasov et al., 1994). Currently in certain areas of the studied lakes, $V$. spiralis forms a mono-species, submerged water meadow up to $2.5 \mathrm{~m}$ in depth. Consequently most native, submerged macrophytes, with the exception of Nuphar, have either moved to deeper parts of the lakes or have disappeared.

An additional alien species Eichhornia crassipes, probably originating from nearby artificial garden ponds, was also noticed at the station on Lake Slesińskie. Eichhornia did not occur at this station throughout the whole year. During the summer months, however, it was checked for ciliates as another potential alien species that may adjust to living there in the future. The native macrophytes such as Ceratophyllum demersum L., Myriophyllum spicatum L., Potamogeton perfoliatus L., Najas marina L., Typha angustifolia L., Nuphar lutea (L.), Sparganium sp., Phragmites australis (Cav.), and Acorus calamus L. were also checked for their ciliate composition.

Sampling was carried out over a 2-month period during the macrophyte vegetation period in 2005. The periphyton samples were taken from submerged parts of macrophytes using a glass tube ( $36 \mathrm{~mm}$ in diameter, $26 \mathrm{~cm}$ long, approximately $210 \mathrm{ml}$ in volume), which was carefully placed on the leaves or shoots of the chosen plant. The top of the tube was closed with a cork. A sample of approximately $20 \mathrm{~cm}$ of the macrophyte, i. e. leaves of submerged macrophytes or stems of emergent macrophytes, was cut, and the bottom of the tube was closed with another cork. The number of samples taken from Vallisneria spiralis and other macrophytes reflect the frequency of occurrence of the plant at the sampling stations. In total, 38 samples from V. spiralis, nine from Myriophyllum, five from Typha, four from Ceratophyllum, three from Najas and Nuphar, and one sample from each of the remaining plants were taken. Among the three lakes 36 samples were taken from Lake Licheńskie, 12 from Lake Mikorzyńskie and 19 from Lake Ślesińskie.

All ciliated protozoa were identified by examining them in vivo under a microscope at appropriate magnification, although when necessary silver staining methods were applied in order to reveal the infraciliature, silverline system and other argentophilic features (Wilbert, 1975; Song, Wilbert, 1995).

Species identification was based on Kahl (1930, 1931, 1932, 1935), Foissner and Berger (1996) and Foissner et al. (1991, 1992, 1994, 1995). Nomenclature is according to Lynn (2008). The allocation of ciliate species to main feeding groups was according to Foissner et al., works.

The faunistic similarities among studied macrophytes were calculated by means of Jaccard's method. For statistical analysis the programs STATISTICA 8.0 and PAST 1.81 (Hammer et al., 2001) were used. 


\section{Results}

During the study, 150 ciliated protozoa taxa in total, belonging to 27 orders and 84 genera, were found on submerged and emergent macrophytes. Among these, 53 species were known as typically periphytic, 81 species occurred mostly in benthos, and 16 were considered to be planktonic. Judging by the species frequency on macrophytes, 133 ciliates belonged to ubiquitous species (more than five records during the study), and 17 species were considered rare (table 1 ).

The average number of ciliate species varied depending on macrophytes (fig. 1). The highest number (20) of ciliate taxa was found on Eichhornia. On Ceratophyllum $18 \pm 3.5$ (mean, SD), and on Myriophyllum, an average of $16 \pm 8.1$ (mean, SD) taxa occurred. On $V$. spiralis, $16 \pm 7.3$ (mean, SD) species of ciliated protozoa were found. Surfaces of Potamogeton, Najas, Typha, Nuphar, Sparganium, and Acorus were less rich in ciliate taxa: on average, 9 to 11 species were found on them. Among emergent macrophytes, only on Phragmites the number of ciliate taxa was higher (15 species). The differences in species number were not significant $(\mathrm{p}>0.05)$.

On Myriophyllum and Vallisneria, 24 and 25 orders of ciliated protozoa were found respectively, but representatives of only 9 orders occurred on Phragmites. The crawling Urostylida, Sporadotrichida and Euplotida dominated on Najas (31\%) and on Ceratophyllum, Myriophyllum, and Acorus (27\% each). Free-swimming Prorodontida were most abundant on Sparganium (31\%) and Acorus (28\%). The stems of Phragmites were dominated by Sessilida and small Philasterida (33\% each). Ciliate species compo-

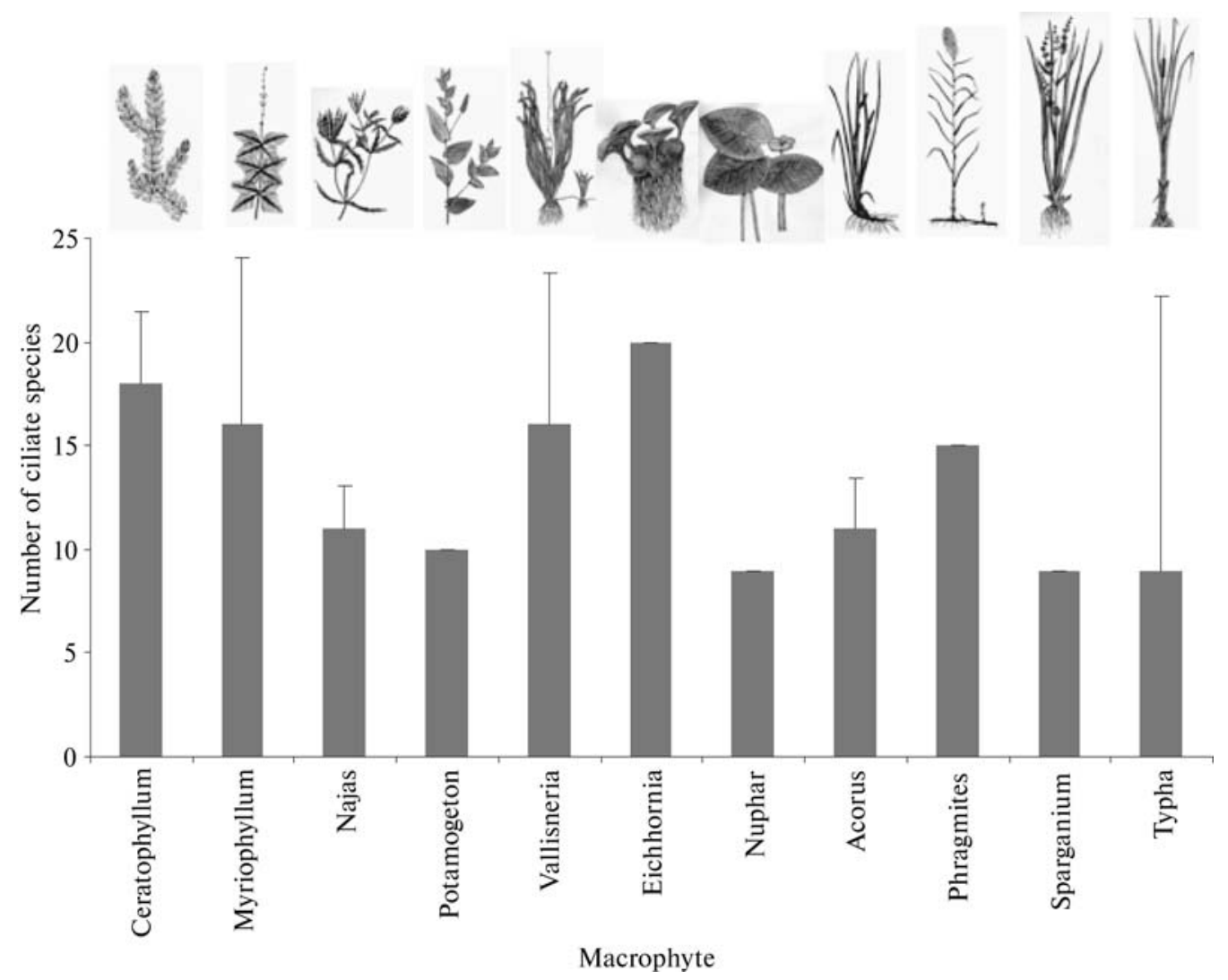

Fig. 1. Mean number of ciliate species found on different macrophytes (mean, SD, * lack of replications).

Рис. 1. Среднее количество видов ресничных простейших, обнаруженное на различных макрофитах (среднее, стандартное отклонение, *повторности отсутствуют). 
Table 1. List of ciliated protozoa and their frequencies (\% of samples) found on Vallisneria spiralis and other macrophytes in littoral zone of Konińskie Lakes (*according to Foissner. Berger, 1996, A - periphyton, B benthos, Fs - anaerobic, $\mathrm{P}$ - planktonic, $\mathrm{T}$ - epizoic).

Таблица 1. Список ресничных простейших и их встречаемость (\% проб) на Vallisneria spiralis и других макрофитах в литоральной зоне Конинских озер (*по: Foissner, Berger, 1996, А - перифитон, В бентос, Fs - анаэробы, P - планктнеры, T - эпизоонты).

\begin{tabular}{|c|c|c|c|c|c|c|c|}
\hline \multirow[b]{2}{*}{ Taxon } & \multicolumn{2}{|c|}{\begin{tabular}{|l|} 
Licheńskie L. \\
\end{tabular}} & \multicolumn{2}{|c|}{ Slesińskie L. } & \multicolumn{2}{|c|}{ Mikorzyńskie L. } & \multirow[b]{2}{*}{$\begin{array}{l}\text { Preffered } \\
\text { habitat* }\end{array}$} \\
\hline & $\begin{array}{c}\text { Vallis- } \\
\text { neria }\end{array}$ & \begin{tabular}{|c|} 
Other \\
macro- \\
phytes
\end{tabular} & $\begin{array}{l}\text { Vallis- } \\
\text { neria }\end{array}$ & $\begin{array}{c}\text { Other } \\
\text { macro- } \\
\text { phytes }\end{array}$ & $\begin{array}{c}\text { Vallis- } \\
\text { neria }\end{array}$ & $\begin{array}{c}\text { Other } \\
\text { macro- } \\
\text { phytes }\end{array}$ & \\
\hline \multicolumn{8}{|l|}{ LOXODIDA JANKOWSKI, 1980} \\
\hline Loxodes striatus (Engelmann,1862) & 0 & 0 & 0 & 0 & 9 & 8 & $\mathrm{~B}, \mathrm{P}$ \\
\hline \multicolumn{8}{|l|}{ HETEROTRICHIDA STEIN, 1859} \\
\hline Spirostomum teres Claparéde \& Lachmann, 1858 & 0 & 3 & 0 & 0 & 0 & 0 & $\mathrm{P}$ \\
\hline Stentor coeruleus (Pallas, 1766) & 22 & 17 & 0 & 5 & 18 & 17 & $\mathrm{~B}, \mathrm{~A}, \mathrm{P}$ \\
\hline Stentor igneus Ehrenberg, 1838 & 17 & 19 & 14 & 26 & 9 & 17 & $\mathrm{~B}, \mathrm{P}$ \\
\hline Stentor muelleri Ehrenberg, 1831 & 0 & 0 & 0 & 5 & 9 & 8 & A \\
\hline Stentor multiformis (Mueller, 1786) & 0 & 0 & 0 & 21 & 0 & 0 & $\mathrm{~B}, \mathrm{~A}$ \\
\hline Stentor polymorphus (Mueller, 1773) & 17 & 11 & 0 & 0 & 0 & 0 & $\mathrm{~B}, \mathrm{~A}$ \\
\hline Stentor roeseli Ehrenberg, 1835 & 30 & 28 & 14 & 42 & 18 & 17 & $\mathrm{~B}, \mathrm{~A}$ \\
\hline \multicolumn{8}{|l|}{ EUPLOTIDA SMALL \& LYNN, 1985} \\
\hline Aspidisca cicada (Mueller, 1786) & 43 & 36 & 29 & 42 & 36 & 33 & $\mathrm{~B}, \mathrm{~A}$ \\
\hline Aspidisca lynceus (Mueller, 1773) & 13 & 11 & 14 & 16 & 0 & 0 & $\mathrm{~B}, \mathrm{~A}$ \\
\hline Euplotes affinis (Dujardin, 1841) & 13 & 14 & 0 & 5 & 9 & 8 & $\mathrm{~B}$ \\
\hline Euplotes moebiusi Kahl, 1932 & 4 & 6 & 0 & 0 & 9 & 8 & $\mathrm{~B}, \mathrm{~A}$ \\
\hline Euplotes patella (Mueller, 1773) & 26 & 33 & 29 & 42 & 27 & 25 & $\mathrm{~B}, \mathrm{~A}$ \\
\hline Euplotes sp. & 0 & 0 & 0 & 5 & 0 & 0 & $\mathrm{~B}, \mathrm{~A}$ \\
\hline \multicolumn{8}{|l|}{ TINTINNIIDA KOFOID \& CAMPBELL, 1929} \\
\hline Codonella cratera (Leidy, 1877) & 9 & 8 & 14 & 11 & 0 & 0 & $\mathrm{P}, \mathrm{B}$ \\
\hline Tintinnidium semiciliatum (Sterki, 1879) & 4 & 3 & 14 & 5 & 0 & 0 & A, B \\
\hline Tintinnidium sp. & 0 & 0 & 14 & 5 & 0 & 0 & $\mathrm{P}$ \\
\hline \multicolumn{8}{|l|}{ CHOREOTRICHIDA SMALL \& LYNN, 1985} \\
\hline Rimostrombidium velox (Faure-Fremiet, 1924) & 0 & 0 & 14 & 5 & 0 & 0 & $\mathrm{P}, \mathrm{B}$ \\
\hline Strobilidium caudatum (Fromentel, 1876) & 0 & 3 & 14 & 11 & 9 & 8 & $\mathrm{P}, \mathrm{B}$ \\
\hline Strobilidium humile Penard, 1922 & 0 & 0 & 14 & 5 & 0 & 0 & $\mathrm{P}, \mathrm{B}$ \\
\hline Strobilidium sp. & 9 & 6 & 0 & 0 & 18 & 17 & $\mathrm{P}$ \\
\hline \multicolumn{8}{|l|}{ STICHOTRICHIDA FAURE-FREMIET, 1961} \\
\hline Kerona pediculus (Mueller, 1773) & 4 & 8 & 0 & 5 & 18 & 17 & $\mathrm{~T}, \mathrm{P}$ \\
\hline \multicolumn{8}{|l|}{ SPORADOTRICHIDA FAURE-FREMIET, 1961} \\
\hline Gonostomum affine Stein, 1859 & 9 & 8 & 0 & 0 & 0 & 0 & $\mathrm{~A}, \mathrm{~B}$ \\
\hline Halteria grandinella (Mueller, 1773) & 13 & 8 & 0 & 0 & 9 & 17 & $\mathrm{P}$ \\
\hline Oxytricha bifaria Stokes, 1887 & 9 & 11 & 43 & 37 & 36 & 33 & $\mathrm{~B}$ \\
\hline Oxytricha sp. & 13 & 17 & 14 & 16 & 9 & 8 & $\mathrm{~B}$ \\
\hline Paraurostyla weissei (Stein, 1859) & 4 & 6 & 0 & 0 & 0 & 0 & $\mathrm{~B}$ \\
\hline Sporadotrichida Gen. sp. & 4 & 3 & 0 & 0 & 0 & 0 & B \\
\hline Stylonychia mytilus Ehrenberg, 1838 & 65 & 56 & 71 & 58 & 36 & 33 & $\mathrm{~B}, \mathrm{~A}$ \\
\hline Stylonychia pustulata (Mueller, 1786) & 22 & 19 & 14 & 11 & 0 & 0 & $\mathrm{~B}, \mathrm{~A}$ \\
\hline Stylonychia putrina Stokes, 1885 & 4 & 3 & 0 & 0 & 0 & 0 & B \\
\hline Tachysoma pellionellum (Mueller, 1773) & 35 & 28 & 43 & 47 & 18 & 17 & $\mathrm{~B}$ \\
\hline \multicolumn{8}{|l|}{ UROSTYLIDA JANKOWSKI, 1979} \\
\hline Diaxonella $\mathrm{sp}$. & 4 & 6 & 0 & 5 & 0 & 8 & $\mathrm{~B}$ \\
\hline Holosticha monilata Kahl, 1928 & 17 & 14 & 0 & 0 & 0 & 0 & $\mathrm{~B}$ \\
\hline Holosticha pullaster (Mueller, 1773) & 22 & 22 & 29 & 32 & 45 & 42 & $\mathrm{~B}$ \\
\hline Holosticha sp. & 17 & 11 & 0 & 0 & 18 & 17 & B \\
\hline Paruroleptus caudatus Stokes, 1886 & 4 & 3 & 14 & 5 & 0 & 0 & $\mathrm{~B}$ \\
\hline Uroleptus musculus (Kahl, 1932) & 4 & 6 & 14 & 11 & 27 & 33 & $\mathrm{~B}, \mathrm{~A}$ \\
\hline Uroleptus piscis (Mueller, 1773) & 0 & 0 & 14 & 11 & 0 & 0 & $\mathrm{~B}, \mathrm{~A}$ \\
\hline Uroleptus sp. & 13 & 8 & 0 & 5 & 0 & 0 & $\mathrm{~B}, \mathrm{~A}$ \\
\hline Urostyla grandis Ehrenberg, 1830 & 17 & 17 & 0 & 5 & 0 & 0 & $\mathrm{~B}$ \\
\hline Urostyla sp. & 0 & 0 & 0 & 0 & 9 & 8 & $\mathrm{~B}$ \\
\hline \multicolumn{8}{|l|}{ STROMBIDIIDA PETZ \& FOISSNER, 1992} \\
\hline Limnostrombidium viride (Stein, 1867) & 9 & 6 & 0 & 5 & 0 & 0 & $\mathrm{P}$ \\
\hline Strombidium sp. & 4 & 3 & 0 & 0 & 9 & 8 & A, B \\
\hline
\end{tabular}


Table 1 (continued).

Продолжение таблицы 1.

\begin{tabular}{|c|c|c|c|c|c|c|c|}
\hline \multirow[b]{2}{*}{ Taxon } & \multicolumn{2}{|c|}{\begin{tabular}{|l|} 
Licheńskie L. \\
\end{tabular}} & \multicolumn{2}{|c|}{ Slesińskie L. } & \multicolumn{2}{|c|}{ Mikorzyńskie L. } & \multirow[b]{2}{*}{$\begin{array}{l}\text { Preffered } \\
\text { habitat* }\end{array}$} \\
\hline & $\begin{array}{c}\text { Vallis- } \\
\text { neria }\end{array}$ & \begin{tabular}{|c|} 
Other \\
macro- \\
phytes
\end{tabular} & $\begin{array}{l}\text { Vallis- } \\
\text { neria }\end{array}$ & $\begin{array}{c}\text { Other } \\
\text { macro- } \\
\text { phytes }\end{array}$ & $\begin{array}{c}\text { Vallis- } \\
\text { neria }\end{array}$ & $\begin{array}{c}\text { Other } \\
\text { macro- } \\
\text { phytes }\end{array}$ & \\
\hline Chaenea sp. & 4 & 3 & 0 & 0 & 0 & 0 & $\mathrm{~B}, \mathrm{~A}$ \\
\hline Enchelyodon fusidens Kahl, 1930 & 0 & 0 & 14 & 11 & 9 & 8 & $\mathrm{~B}, \mathrm{~A}$ \\
\hline Enchelys gasterosteus (Kahl, 1926) & 0 & 0 & 0 & 0 & 0 & 8 & Fs, B \\
\hline Homalozoon vermiculare (Stokes, 1887) & 4 & 3 & 0 & 0 & 9 & 8 & $\mathrm{~B}, \mathrm{~A}$ \\
\hline Lacrymaria filiformis Maskell, 1886 & 0 & 0 & 0 & 0 & 0 & 8 & $\mathrm{~B}, \mathrm{~A}$ \\
\hline Lacrymaria olor (Mueller, 1786) & 4 & 8 & 0 & 11 & 0 & 0 & $\mathrm{~B}, \mathrm{~A}$ \\
\hline Phialina minima (Kahl, 1927) & 4 & 6 & 0 & 0 & 0 & 0 & $\mathrm{~B}, \mathrm{~A}$ \\
\hline Spathidium sp. & 4 & 3 & 0 & 0 & 9 & 8 & $\mathrm{~A}, \mathrm{~B}, \mathrm{P}$ \\
\hline Trachelius ovum (Ehrenberg, 1831) & 9 & 11 & 14 & 11 & 0 & 0 & $\mathrm{~A}, \mathrm{~B}, \mathrm{P}$ \\
\hline Trachelophyllum apiculatum (Perty, 1852) & 4 & 3 & 0 & 0 & 0 & 0 & $\mathrm{~A}, \mathrm{~B}$ \\
\hline \multicolumn{8}{|l|}{ PLEUROSTOMATIDA SCHEWIAKOFF, 1896} \\
\hline Acineria incurvata Dujardin, 1841 & 0 & 0 & 0 & 5 & 0 & 0 & $\mathrm{~A}, \mathrm{~B}$ \\
\hline Acineria uncinata Tucolesco, 1962 & 48 & 31 & 29 & 26 & 36 & 33 & $\mathrm{~A}, \mathrm{~B}$ \\
\hline Amphileptus pleurosigma (Stokes, 1884) & 22 & 14 & 14 & 11 & 0 & 0 & A, B \\
\hline Amphileptus procerus (Penard, 1922) & 4 & 8 & 14 & 26 & 18 & 17 & $\mathrm{~B}$ \\
\hline Amphileptus sp. & 0 & 0 & 0 & & 9 & 8 & $\mathrm{~A}, \mathrm{~B}$ \\
\hline Litonotus anguilla Kahl, 1930 & 0 & 0 & 0 & 5 & 0 & 0 & $\mathrm{~B}, \mathrm{~A}$ \\
\hline Litonotus crystallinus (Vuxanovici, 1960) & 0 & 3 & 14 & 5 & 0 & 0 & $\mathrm{~B}, \mathrm{~A}$ \\
\hline Litonotus cygnus (Mueller, 1773) & 9 & 14 & 0 & 11 & 0 & 0 & $\mathrm{~B}, \mathrm{~A}$ \\
\hline Litonotus lamella (Mueller, 1773) & 17 & 22 & 14 & 16 & 18 & 17 & $\mathrm{~B}, \mathrm{~A}$ \\
\hline Loxophyllum helus (Stokes, 1884) & 9 & 6 & 0 & 0 & 0 & 0 & $\mathrm{~A}, \mathrm{~B}$ \\
\hline Loxophyllum meleagris (Mueller, 1773) & 13 & 14 & 0 & 5 & 9 & 8 & $\mathrm{~A}, \mathrm{~B}$ \\
\hline \multicolumn{8}{|l|}{ CHLAMYDODONTIDA DEROUX, 1976} \\
\hline Chilodonella uncinata (Ehrenberg, 1838) & 48 & 39 & 29 & 32 & 18 & 17 & $\mathrm{~A}, \mathrm{~B}$ \\
\hline Chlamydodon sp. & 0 & 3 & 0 & 0 & 0 & 0 & A, B \\
\hline Gastronauta membranaceus Buetschli, 1889 & 0 & 0 & 14 & 5 & 9 & 8 & A, B \\
\hline Pseudochilodontopsis fluviatilis Foissner, 1988 & 0 & 3 & 0 & 0 & 0 & 0 & A, B \\
\hline Pseudochilodontopsis sp. & 4 & 3 & 0 & 5 & 9 & 8 & A, B \\
\hline Trithigmostoma cucullulus (Mueller, 1786) & 9 & 14 & 0 & 5 & 45 & 42 & A, B \\
\hline \multicolumn{8}{|l|}{ DYSTERIIDA DEROUX, 1976} \\
\hline Dysteria fluviatilis (Stein, 1859) & 4 & 6 & 0 & 11 & 0 & 0 & $\mathrm{~A}, \mathrm{~B}$ \\
\hline Trochilia minuta (Roux, 1899) & 17 & 14 & 14 & 21 & 27 & 25 & $\mathrm{~A}, \mathrm{~B}$ \\
\hline \multicolumn{8}{|l|}{ ENDOGENIDA COLLIN, 1912} \\
\hline Acineta tuberosa Ehrenberg, 1833 & 0 & 0 & 0 & 5 & 0 & 0 & $\mathrm{~A}, \mathrm{~T}$ \\
\hline \multicolumn{8}{|l|}{ SYNHYMENIIDA PUYTORAC ET AL. } \\
\hline $\begin{array}{l}\text { IN DEROUX, 1978 } \\
\text { Chilodontopsis depressa (Perty, 1852) }\end{array}$ & 9 & 14 & 0 & 0 & 0 & 0 & A, B \\
\hline Zosterodasys transversa (Kahl, 1928) & 0 & 3 & 0 & 0 & 0 & 0 & A, B \\
\hline \multicolumn{8}{|l|}{ NASSULIDA JANKOWSKI, 1967} \\
\hline Furgasonia trichocystis (Stokes, 1894) & 0 & 3 & 0 & 0 & 0 & 0 & $\mathrm{P}$ \\
\hline Nassula picta Greeff, 1888 & 0 & 3 & 0 & 0 & 9 & 8 & $\mathrm{~B}, \mathrm{~A}, \mathrm{P}$ \\
\hline Nassula sp. & 0 & 0 & 0 & 0 & 9 & 8 & $\mathrm{~B}, \mathrm{~A}, \mathrm{P}$ \\
\hline Obertrumia aurea (Ehrenberg, 1833) & 0 & 3 & 14 & 11 & 9 & 8 & $\mathrm{~B}, \mathrm{P}$ \\
\hline \multicolumn{8}{|l|}{ MICROTHORACIDA JANKOWSKI, 1967} \\
\hline Pseudomicrothorax sp. & 0 & 0 & 0 & 0 & 9 & 8 & $\mathrm{~A}, \mathrm{~B}$ \\
\hline \multicolumn{8}{|l|}{ BURSARIOMORPHIDA FERNANDEZ- } \\
\hline Bursaridium pseudobursaria (Faure-Fremiet, 1924) & 0 & 0 & 0 & 5 & 0 & 0 & $P$ \\
\hline \multicolumn{8}{|l|}{ CYRTOLOPHOSIDIDA FOISSNER, 1978} \\
\hline Cyrtolophosis mucicola Stokes, 1885 & 0 & 0 & 0 & 0 & 18 & 17 & $\mathrm{~B}$ \\
\hline \multicolumn{8}{|l|}{ PRORODONTIDA CORLISS, 1974} \\
\hline Coleps hirtus (Mueller, 1786) & 74 & 69 & 57 & 53 & 64 & 67 & $\mathrm{~A}, \mathrm{~B}, \mathrm{P}$ \\
\hline Coleps spetai Foissner, 1984 & 26 & 19 & 0 & 5 & 18 & 17 & $\mathrm{P}$ \\
\hline Holophrya discolor Ehrenberg, 1833 & 9 & 8 & 29 & 16 & 9 & 8 & $\mathrm{~B}, \mathrm{P}$ \\
\hline Holophrya teres Ehrenberg, 1833 & 4 & 6 & 0 & 0 & 0 & 0 & $\mathrm{~B}, \mathrm{P}$ \\
\hline Placus luciae (Kahl, 1926) & 4 & 3 & 0 & 0 & 0 & 8 & $\mathrm{~B}, \mathrm{~A}$ \\
\hline Prorodon niveus Ehrenberg, 1833 & 0 & 0 & 0 & 5 & 0 & 0 & $\mathrm{~B}$ \\
\hline
\end{tabular}


Table 1 (continued).

Продолжение таблицы 1.

\begin{tabular}{|c|c|c|c|c|c|c|c|}
\hline \multirow[b]{2}{*}{ Taxon } & \multicolumn{2}{|c|}{ Licheńskie L. } & \multicolumn{2}{|c|}{ Ślesińskie L. } & \multicolumn{2}{|c|}{ Mikorzyńskie L. } & \multirow[b]{2}{*}{$\begin{array}{l}\text { Preffered } \\
\text { habitat* }\end{array}$} \\
\hline & $\begin{array}{c}\text { Vallis- } \\
\text { neria }\end{array}$ & $\begin{array}{c}\text { Other } \\
\text { macro- } \\
\text { phytes }\end{array}$ & $\begin{array}{c}\text { Vallis- } \\
\text { neria }\end{array}$ & $\begin{array}{c}\text { Other } \\
\text { macro- } \\
\text { phytes }\end{array}$ & $\begin{array}{c}\text { Vallis- } \\
\text { neria }\end{array}$ & $\begin{array}{c}\text { Other } \\
\text { macro- } \\
\text { phytes }\end{array}$ & \\
\hline Urotricha agilis (Stokes, 1886) & 0 & 3 & 0 & 0 & 0 & 0 & $\mathrm{~B}, \mathrm{P}$ \\
\hline Urotricha armata Kahl, 1927 & 4 & 8 & 0 & 0 & 0 & 0 & $\mathrm{~B}, \mathrm{~A}$ \\
\hline Urotricha furcata Schewiakoff, 1892 & 4 & 6 & 0 & 0 & 9 & 8 & $\mathrm{P}$ \\
\hline Urotricha ovata Kahl, 1926 & 13 & 11 & 0 & 5 & 9 & 8 & $\mathrm{~B}, \mathrm{P}$ \\
\hline Urotricha sp. & 13 & 11 & 0 & 0 & 9 & 8 & $\mathrm{~B}, \mathrm{P}$ \\
\hline \multicolumn{8}{|l|}{ PENICULIDA FAURE-FREMIET } \\
\hline \multicolumn{8}{|l|}{ IN CORLISS, 1956} \\
\hline Frontonia acuminata (Ehrenberg, 1833) & 13 & 11 & 14 & 11 & 18 & 17 & $\mathrm{~B}, \mathrm{~A}, \mathrm{P}$ \\
\hline Frontonia angusta Kahl, 1931 & 9 & 11 & 0 & 5 & 18 & 25 & $\mathrm{~B}, \mathrm{~A}, \mathrm{P}$ \\
\hline Frontonia atra (Ehrenberg, 1833) & 9 & 11 & 0 & 0 & 9 & 8 & $\mathrm{~B}, \mathrm{P}$ \\
\hline Frontonia leucas (Ehrenberg, 1833) & 0 & 0 & 0 & 5 & 0 & 0 & $\mathrm{~B}, \mathrm{~A}, \mathrm{P}$ \\
\hline Frontonia roquei Dragesco, 1970 & 9 & 6 & 0 & 0 & 9 & 8 & $\mathrm{~B}, \mathrm{~A}, \mathrm{P}$ \\
\hline Lembadion lucens (Maskell, 1887) & 4 & 14 & 0 & 0 & 0 & 0 & $\mathrm{~B}, \mathrm{P}$ \\
\hline Paramecium aurelia complex Sonneborn, 1975 & 4 & 8 & 0 & 0 & 0 & 0 & $\mathrm{~B}, \mathrm{P}$ \\
\hline Paramecium bursaria (Ehrenberg, 1831) & 9 & 6 & 0 & 0 & 0 & 0 & $\mathrm{~A}, \mathrm{~B}, \mathrm{P}$ \\
\hline Paramecium caudatum Ehrenberg, 1833 & 9 & 8 & 0 & 0 & 9 & 8 & $\mathrm{~B}, \mathrm{~A}, \mathrm{P}$ \\
\hline Stokesia vernalis Wenrich, 1929 & 0 & 3 & 0 & 0 & 0 & 0 & $\mathrm{P}$ \\
\hline Urocentrum turbo (Mueller, 1876) & 4 & 3 & 0 & 0 & 0 & 0 & $\mathrm{~B}, \mathrm{~A}, \mathrm{P}$ \\
\hline \multicolumn{8}{|l|}{ PHILASTERIDA SMALL, 1967} \\
\hline Cinetochilum margaritaceum (Ehrenberg, 1831) & 35 & 39 & 43 & 53 & 55 & 58 & $\mathrm{~A}, \mathrm{~B}, \mathrm{P}$ \\
\hline Dexiotricha granulosa (Kent, 1881) & 9 & 6 & 14 & 5 & 0 & 8 & $\mathrm{~B}, \mathrm{~A}$ \\
\hline Philasterida Gen. sp. 1 & 0 & 0 & 0 & 11 & 18 & 17 & $\mathrm{~B}, \mathrm{P}$ \\
\hline Philasterida Gen. sp. 2 & 0 & 0 & 0 & 5 & 9 & 8 & $\mathrm{~B}, \mathrm{P}$ \\
\hline Philasterida Gen. sp. 3 & 0 & 0 & 14 & 5 & 9 & 8 & $\mathrm{~B}, \mathrm{P}$ \\
\hline Pseudocohnilembus pusillus (Quennerstedt, 1869) & 13 & 19 & 14 & 16 & 64 & 67 & $\mathrm{~B}, \mathrm{P}$ \\
\hline Uronema nigricans (Mueller, 1786) & 26 & 22 & 14 & 5 & 0 & 8 & $\mathrm{~B}, \mathrm{~A}, \mathrm{P}$ \\
\hline Urozona buetschlii Schewiakoff, 1889 & 0 & 3 & 0 & 0 & 0 & 0 & $\mathrm{~B}, \mathrm{P}$ \\
\hline \multicolumn{8}{|l|}{ PLEURONEMATIDA FAURE-FREMIET } \\
\hline \multicolumn{8}{|l|}{ IN CORLISS, 1956} \\
\hline Ctedoctema acanthocryptum Stokes, 1884 & 13 & 17 & 0 & 11 & 73 & 75 & B \\
\hline Cyclidium glaucoma Mueller, 1773 & 48 & 42 & 29 & 32 & 64 & 67 & $\mathrm{~B}, \mathrm{~A}, \mathrm{P}$ \\
\hline Cyclidium versatile Penard, 1922 & 0 & 0 & 0 & 5 & 27 & 25 & $\mathrm{~B}, \mathrm{~A}, \mathrm{P}$ \\
\hline Cyclidium sp. & 4 & 8 & 0 & 5 & 0 & 0 & $\mathrm{~B}, \mathrm{~A}, \mathrm{P}$ \\
\hline Pleuronema coronatum Kent, 1881 & 0 & 3 & 0 & 0 & 9 & 8 & $\mathrm{~B}$ \\
\hline \multicolumn{8}{|l|}{ TETRAHYMENIDA FAURE-FREMIET } \\
\hline \multicolumn{8}{|l|}{ IN CORLISS, 1956} \\
\hline Colpidium colpoda (Losana, 1829) & 0 & 3 & 0 & 0 & 0 & 0 & B \\
\hline Dexiostoma campylum (Stokes, 1886) & 4 & 3 & 0 & 0 & 0 & 0 & B \\
\hline \multicolumn{8}{|l|}{ Tetrahymena pyriformis complex } \\
\hline Nanney\& McCoy, 1976 & 22 & 17 & 14 & 26 & 9 & 8 & B \\
\hline \multicolumn{8}{|l|}{ OPHRYOGLENIDA CANELLA, 1964} \\
\hline Ophryoglena flava (Ehrenberg, 1833) & 13 & 11 & 0 & 11 & 9 & 8 & B \\
\hline Ophryoglena utriculariae Kahl, 1930 & 0 & 3 & 0 & 0 & 0 & 0 & $\mathrm{~B}$ \\
\hline \multicolumn{8}{|l|}{ SESSILIDA KAHL, 1933} \\
\hline Campanella umbellaria (Linnaeus, 1758) & 4 & 3 & 0 & 5 & 0 & 0 & $\mathrm{~A}, \mathrm{~B}, \mathrm{~T}$ \\
\hline Carchesium polypinum (Linnaeus, 1758) & 4 & 6 & 0 & 0 & 0 & 0 & $\mathrm{~B}, \mathrm{~A}, \mathrm{~T}$ \\
\hline Cothurnia sp. & 0 & 0 & 14 & 5 & 9 & 8 & $\mathrm{~A}, \mathrm{~B}, \mathrm{~T}$ \\
\hline Epistylis chrysemydis Bishop \& Jahn, 1941 & 0 & 0 & 14 & 5 & 0 & 0 & $\mathrm{~A}, \mathrm{~T}$ \\
\hline Epistylis keronata Nusch, 1970 & 0 & 0 & 0 & 0 & 27 & 25 & $\mathrm{~A}, \mathrm{~T}$ \\
\hline Epistylis hentscheli Kahl, 1935 & 4 & 8 & 14 & 5 & 0 & 0 & A, B \\
\hline Epistylis plicatilis Ehrenberg, 1831 & 0 & 0 & 0 & 0 & 9 & 8 & $\mathrm{~A}, \mathrm{~B}, \mathrm{~T}$ \\
\hline Opercularia articulata Goldfuss, 1820 & 0 & 0 & 0 & 0 & 18 & 17 & $\mathrm{~A}, \mathrm{~T}$ \\
\hline Opercularia nutans (Ehrenberg, 1831) & 17 & 14 & 0 & 0 & 0 & 0 & $\mathrm{~A}, \mathrm{~T}$ \\
\hline Platycola decumbens (Ehrenberg, 1830) & 0 & 0 & 0 & 11 & 0 & 0 & A \\
\hline Pyxicola sp. & 0 & 0 & 0 & 0 & 9 & 8 & A \\
\hline Sessilida Gen. sp. & 0 & 0 & 0 & 0 & 18 & 17 & A, B \\
\hline Thuricola folliculata Kent,1881 & 0 & 0 & 0 & 11 & 0 & 0 & A \\
\hline
\end{tabular}


Table 1 (continued).

Окончание таблицы 1.

\begin{tabular}{|c|c|c|c|c|c|c|c|}
\hline \multirow[b]{2}{*}{ Taxon } & \multicolumn{2}{|c|}{\begin{tabular}{|l|} 
Licheńskie L. \\
\end{tabular}} & \multicolumn{2}{|c|}{ Slesińskie L. } & \multicolumn{2}{|c|}{ Mikorzyńskie L. } & \multirow[b]{2}{*}{$\begin{array}{l}\text { Preffered } \\
\text { habitat* }\end{array}$} \\
\hline & $\begin{array}{c}\text { Vallis- } \\
\text { neria }\end{array}$ & $\begin{array}{c}\text { Other } \\
\text { macro- } \\
\text { phytes }\end{array}$ & $\begin{array}{c}\text { Vallis- } \\
\text { neria }\end{array}$ & $\begin{array}{l}\text { Other } \\
\text { macro- } \\
\text { phytes }\end{array}$ & $\begin{array}{l}\text { Vallis- } \\
\text { neria }\end{array}$ & $\begin{array}{c}\text { Other } \\
\text { macro- } \\
\text { phytes }\end{array}$ & \\
\hline Thuricola kellicottiana (Stokes, 1887) Kahl, 1935 & 4 & 3 & 0 & 0 & 0 & 0 & A \\
\hline Vagnicola ingenita (Mueller, 1786) & 0 & 0 & 0 & 21 & 9 & 8 & A, T \\
\hline Vagnicola sp. & 4 & 3 & 0 & 0 & 0 & 0 & $\mathrm{~A}, \mathrm{~T}$ \\
\hline Vorticella aquadulcis Stokes, 1887 & 4 & 6 & 14 & 21 & 9 & 8 & $\mathrm{~A}, \mathrm{~B}$ \\
\hline Vorticella campanula (Ehrenberg, 1830) & 78 & 78 & 100 & 89 & 73 & 67 & $\mathrm{~A}, \mathrm{~B}, \mathrm{~T}$ \\
\hline Vorticella convallaria Linnaeus, 1767 & 52 & 61 & 14 & 53 & 73 & 75 & $\mathrm{~A}, \mathrm{~B}, \mathrm{~T}$ \\
\hline Vorticella marginata Stiller, 1931 & 4 & 3 & 0 & 0 & 0 & 0 & $\mathrm{~A}, \mathrm{~B}$ \\
\hline Vorticella octava Stokes, 1885 & 0 & 3 & 0 & 0 & 0 & 0 & $\mathrm{~A}, \mathrm{~B}$ \\
\hline Vorticella picta Ehrenberg, 1838 & 4 & 3 & 0 & 0 & 18 & 17 & $\mathrm{~A}, \mathrm{~B}$ \\
\hline Vorticella $\mathrm{sp}$ & 4 & 3 & 0 & 0 & 0 & 0 & $\mathrm{~A}, \mathrm{~B}$ \\
\hline Zoothamnium arbuscula Ehrenberg, 1838 & 9 & 6 & 14 & 5 & 0 & 0 & A \\
\hline Zoothamnium procerius Kahl, 1935 & 4 & 3 & 0 & 0 & 0 & 0 & $\mathrm{~A}, \mathrm{~B}, \mathrm{~T}$ \\
\hline Zoothamnium simplex Kent, 1881 & 4 & 3 & 0 & 5 & 0 & 0 & $\mathrm{~A}, \mathrm{~B}, \mathrm{~T}$ \\
\hline MOBILIDA KAHL, 1933 & & & & & & & \\
\hline Trichodina pediculus Ehrenberg, 1831 & 0 & 0 & 0 & 5 & 9 & 8 & $\mathrm{~T}, \mathrm{P}$ \\
\hline Total number of taxa & 96 & 112 & 48 & $\begin{array}{l}85 \\
150\end{array}$ & 74 & 80 & \\
\hline
\end{tabular}

sition on Potamogeton was not rich, but represented by 10 orders and $14-15 \%$ of total species found.

Representatives of sessile, crawling, and free-swimming ciliates occurred on all macrophytes. On Phragmites and Sparganium, the free-swimming ciliates were dominant (50-68\% of species present), while on Myriophyllum, Ceratophyllum, Acorus, and Najas, the crawling ciliates were the most numerous and reached, respectively, 45, 52 56 , and $59 \%$ of the total species present (fig. 2). The percentage of sessile species var-

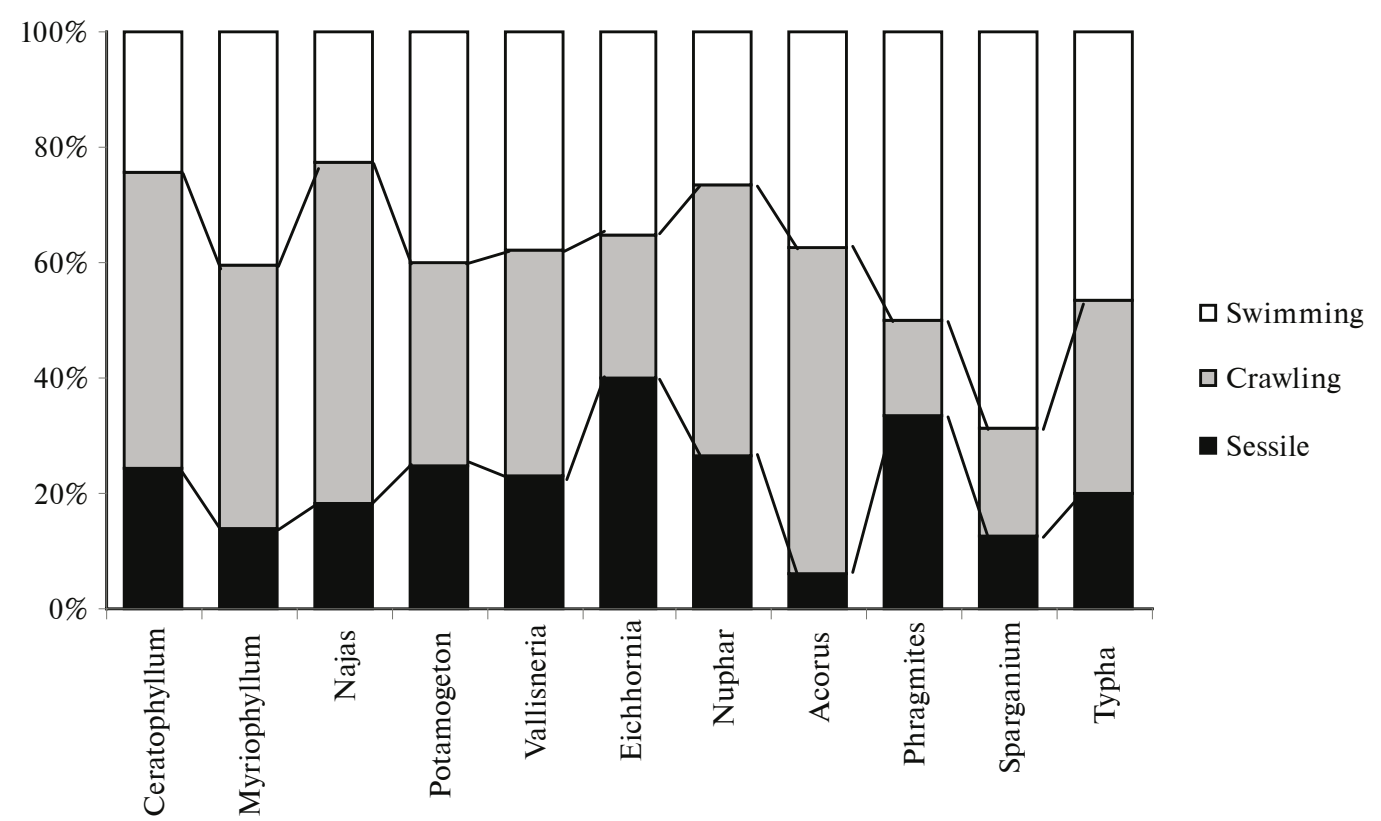

Fig. 2. Percentage of species number of sessile, crawling, and free-swimming ciliates on macrophytes.

Рис. 2. Процентное соотношение количества видов сессильных, ползающих и свободноплавающих цилиат на макрофитах. 


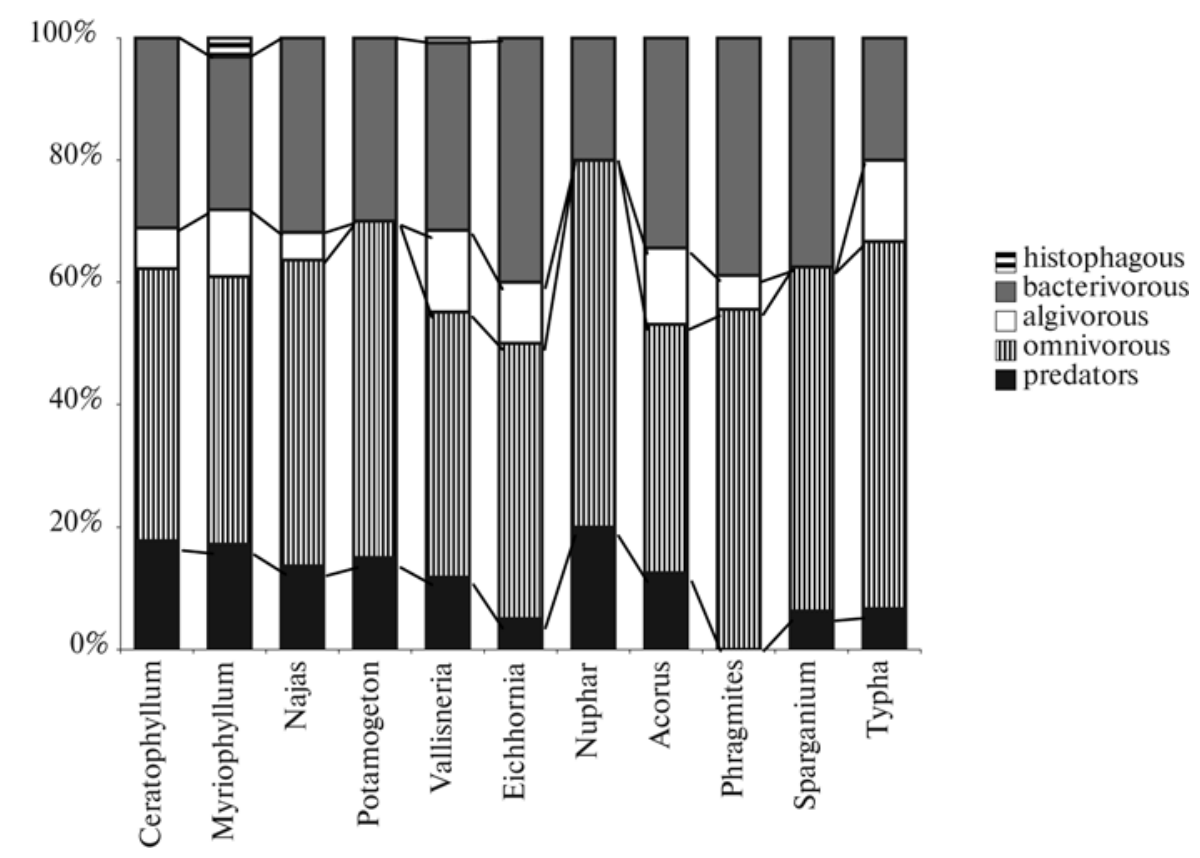

Fig. 3. Percentage of ciliate feeding groups on macrophytes.

Рис. 3. Процентное соотношение различных трофических групп цилиат на макрофитах.

ied among the macrophytes from $6 \%$ on Acorus to $40 \%$ on Eichhornia, which was a result of only two species on Typha, Nuphar, Sparganium, and Acorus and of eight sessile species on Eichhornia. The percentage of crawling ciliates had different pattern. On Najas, Acorus, Ceratophyllum, Nuphar and Myriophyllum, crawling species were dominant and reached from 59\% to $45 \%$ respectively. On Typha, Potamogeton and Vallisneria crawling ciliates accounted for between 33 and $39 \%$ of the total species, and on Sparganium and Phragmites the percentage of crawling ciliate species was less than $20 \%$. Sessile and crawling species considered as typically periphytic were the most abundant on Nuphar, Myriophyllum and Ceratophyllum.

The dominant group of ciliates living on macrophytes were bacterivorous (20$44 \%$ ) or omnivorous (22-40\%). They dominated on Phragmites and Sparganium (66\% and $69 \%$ together, respectively) and on Eichhornia (40\% bacterivorous). Predators comprised between $5 \%$ of ciliate species on Eichhornia to $18 \%$ on Ceratophyllum. Histophagous ciliates were rare and associated only with Myriophyllum (3\%) and V. spi-

Table 2. Similarity of ciliate species composition among Vallisneria spiralis and other macrophytes. Таблица 2. Сходство видового состава цилиат между Vallisneria spiralis и другими макрофитами.

\begin{tabular}{l|c}
\multicolumn{1}{c|}{ Macrophyte } & Jaccard index \\
\hline Eichhornia crassipes (Mart.) & 63,20 \\
Ceratophyllum demersum L. & 52,80 \\
Myriophyllum spicatum L. & 14,80 \\
Potamogeton perfoliatus L. & 5,88 \\
Najas marina L. & 41,67 \\
Typha angustifolia L. & 14,12 \\
Nuphar lutea (L.) & 6,60 \\
Sparganium sp. & 10,39 \\
Phragmites australis (Cav.) & 13,27 \\
Acorus calamus L. & 11,46 \\
\hline
\end{tabular}


ralis (1\%). All five trophic groups were present only on $V$. spiralis and Myriophyllum (fig. 3).

The Jaccard similarity index among ciliate composition on $V$. spiralis and other macrophytes was the highest for the alien form Eichhornia and the native form Ceratophyllum. The lowest similarity was among Vallisneria and native emergent and floating macrophytes (table 2).

\section{Discussion}

The composition of ciliate assemblages depends on habitat, water chemistry (especially nutrients), and surface of substrates. Number of species depends on the methods used, the surfaces studied and the habitat. Coppellotti and Matarazzo (2000), as an example, investigated ciliate colonization of glass slides in the Lagoon of Venice and found 45 species. In saline habitats in Jiaozhou Bay, China, the occurrence of 37 species of ciliates from 10 orders was noted on glass slides used as artificial surfaces (Gong et al., 2005), while 130 species of ciliates were reported on a combination of submerged objects and glass slides in the Caspian Sea (Agamaliev, 1974).

The results reported in the literature demonstrated strong affects of habitat structure and architecture of macrophyte leaf or stem on the spatial distribution and taxonomic composition of aquatic organisms (Duggan et al., 2001; Mieczan, 2007; Pals et al., 2006). Macrophytes strongly influence protozoan species composition by modifying protozoan food availability and increasing the spatial heterogeneity (Biyu, 2000). In a study of a macrophyte-abundant shallow lake in Eastern Poland, Mieczan (2007) found 23 ciliate species on Chara and Ceratophyllum stands and 10-14 species on Phragmites and Typha. In our study, the mean numbers of ciliate species found on Ceratophyllum, Phragmites, and Typha were similar to those reported by Mieczan (2007), i. e. 18, 15, and 9 species, respectively.

Ciliate assemblage on macrophytes in heated lakes is characterized by compex trophic structure composed of algivorous, bacterivorous, predators, omnivorous, and histophagous. Representatives of all trophic groups were found on native Ceratophyllum and Myriophyllum. At the simple surfaces of native macrophytes such as Phragmites or Typha, only the Sessilida and small bacterivorous forms from Prorodontida were abundant.

Although a well-developed periphyton community is supposed to occur on macrophytes with architecturally complicated structures, the results of our study showed that leaves of $V$. spiralis, in spite of their simple architecture, also had a rich ciliate assemblage with compex trophic structure. This is in contrast with the statement that ciliates prefer plants with complicated architecture, but supports the hypothesis that food availability and stable conditions play an important role for the ciliates. However, it should be noted that the dense patches of Vallisneria form peculiar space which is similar to macrophytes with complicated surface.

We found more ciliate species on leaves of the alien $V$. spiralis than on the submerged native macrophytes such as Potamogeton or Najas. This is even more clearly noticeable when compared with native emergent plants. Similar results for invertebrate communities associated with $V$. americana and Euroasian water-chestnut (Trapa natans L.) were reported by Strayer et al. (2003) who demonstrated, that the replacement of the native macrophyte ( $V$. americana) by an alien (Trapa) in the Hudson River caused the increase in macroinvertebrate densities and probably increased the systemwide biodiversity.

The present study confirms that the average number of ciliate species on native macrophytes was higher on architecturally complex submerged forms such as Ceratophyllum, and Myriophyllum than on emergent macrophytes with simple architec- 
ture in their submerged parts such as Acorus, Sparganium, or Typha. In addition our results suggest that the replacement of the native macrophytes by monospecies water meadows of Vallisneria in the littoral of heated Konińskie Lakes did not negatively change the number of species and complexity of ciliate assemblage.

This work was financially supported by the Ministry of Science and Higher Education (project no. 2 P04G 088 26) and by Jagiellonian University DS/WBiNoZ/INoS/756.

Agamaliev F. G. Ciliates of the solid surface overgrowth of the Caspian Sea // Acta Protozoologica. 1974. - 13. - P. 53-83.

Biyu S. Planktonic protozooplankton (ciliates, heliozoans and testaceans) in two shallow mesotrophic lakes in China - a comparative study between a macrophyte-dominated lake (Biandantang) and an algal lake (Houhu) // Hydrobiologia. - 2000. - 434. - P. 151-163.

Coppellotti O., Matarazzo P. Ciliates colonization of artificial substrates in the Lagoon of Venice // Journal of the Marine Biological Association of the UK. - 2000. - 80. - P. 419-427.

Duggan I. C., Green J. D., Thompson K., Shiel R. J. The influence of macrophytes on the spatial distribution of littoral rotifers // Freshwater Biology. - 2001. - 46. - P. 777-786.

Foissner W., Blatterer H., Berger H., Kohmann F. Taxonomische und ökologische Revision der Ciliaten des Saprobiensystems - Band I: Cyrtophorida, Oligotrichida, Hypotrichia, Colpodea. - München : Informationsberichte des Bayer. Landesamtes für Wasserwirtschaft, 1991. - $478 \mathrm{~S}$.

Foissner W., Berger H., Kohmann F. Taxonomische und ökologische Revision der Ciliaten des Saprobiensystems - Band II: Peritrichia, Heterotrichida, Odontostomatida. - München : Informationsberichte des Bayer. Landesamtes für Wasserwirtschaft, 1992. - $502 \mathrm{~S}$.

Foissner W., Berger H., Kohmann F. Taxonomische und ökologische Revision der Ciliaten des Saprobiensystems - Band III: Hymenostomata, Prostomatida, Nassulida. - Informationsberichte des Bayer. Landesamtes für Wasserwirtschaft, München, 1994. - 548 S.

Foissner W., Berger H. , Blatterer H. , Kohmann F. Taxonomische und ökologische Revision der Ciliaten des Saprobiensystems - Band IV: Gymnostomatea, Loxodes, Suctoria. - München : Informationsberichte des Bayer. Landesamtes für Wasserwirtschaft, 1995. - $544 \mathrm{~S}$.

Foissner W., Berger H. A user-friendly guide to the ciliates (Protozoa, Ciliophora) commonly used by hydrobiologists as bioindicators in rivers, lakes, and waste waters, with notes on their ecology // Freshwater Biology. - 1996. - 35. - P. 375-482.

Gąbka M. Vallisneria spiralis (Hydrocharitaceae) - nowy gatunek we florze Polski // Fragmenta Floristica et Geobotanica Polonica. - 2002. - 9. - P. 67-73.

Gong J., Song W., Warren A. Periphytic ciliate colonization: annual cycle and responses to environmental conditions // Aquatic Microbial Ecology. - 2005. - 39. - P. 159-170.

Hammer O., Harper D. A. T., Ryan P. D. PAST: Palaeontological statistics software package for education and data analysis // Paleontologia Electronica. - 2001. - 4. - P. 1-9.

Hutorowicz A., Dziedzic J., Kapusta A. Vallisneria spiralis (Hydrocharitaceae) localities in Konin Lakes (Kujawy Lakeland) // Fragmenta Floristica et Geobotanica Polonica. - 2006. - 13. - P. 9-94.

Kahl A. Urtiere oder Protozoa I: Wimpertiere oder Ciliata (Infusoria) 1. Allgemeiner Teil und Prostomata. Tierwelt Dtl., 18, 1930. - P. 1-180.

Kahl A. Urtiere oder Protozoa I: Wimpertiere oder Ciliata (Infusoria) 2. Holotricha auЯer den im 1. Teil behandelten Prostomata. - Tierwelt Dtl., 21, 1931. - S. 181-398.

Kahl A. Urtiere oder Protozo a I: Wimpertiere oder Ciliata (Infusoria) 3. Spirotricha. - Tierwelt Dtl., 25, 1932. - S. 399-650.

Kahl A. Urtiere oder Protozoa I: Wimpertiere oder Ciliata (Infusoria) 4. Peritricha und Chonotricha. Tierwelt Dtl., 30, 1935. - S. 651-886.

Lynn D. H. The Ciliated Protozoa. Characterization, Classification, and Guide to the Literature. Springer Science+Business Media B. V. - 2008. - 605 p.

Messyasz B. , Kuczyńska-Kippen N. Periphytic algal communities: a comparison on Typha angustifolia L. and Chara tomentosa L. beds in three shallow lakes (West Poland) // Polish Journal of Ecology. 2006. - 54. - P. 15-27.

Mieczan T. Periphytic ciliates in littoral zone of three lakes of different trophic status // Polish Journal of Ecology. - 2005. - 53. - P. 489-02.

Mieczan T. Size spectra and abundance of planktonic ciliates within various habitats in a macrophytedominated lake (Eastern Poland) // Biologia. - 2007. - 62. - P. 189-194.

Pals A., Elst D., Muylaert K., Van Assche J. Substrate specificity of periphytic desmids in shallow softwater lakes in Belgium // Hydrobiologia. - 2006. - 568. - P. 159-168.

Primc-Habdija B., Radanowic J. Seasonal changes in trophic structure of periphytic ciliates in relation to discharge regime // Verh. Internat. Verein. Limnol. - 1998. - 26. - P. 116-119.

Primc-Habdija B., Habdija I., Plenkovic-Moraj A. Tufa deposition and periphyton overgrowth as factors affecting the ciliate community on travertine barriers in different current velocity conditions // Hydrobiologia. - 2001. - 457. - P. 87-96. 
Protasov A. A., Afanasiev S. A., Sinicyna O. O., Zdanowski B. Composition and functioning of benthic communities // Archiwum Rybactwa Polskiego. - 1994. - 2. - P. 257-284.

Raffaelli D., Hall S., Emes C., Manly B. Constraints on body size distributions: an experimental approach using a small-scale system // Oecologia. - 2000. - 122. - P. 89-398.

Sleigh M. A., Baldock B. M., Baker J. H. Protozoan communities in chalk streams // Hydrobiologia. 1992. - 248. - P. 3-64.

Socha D., Zdanowski B. Ekosystemy wodne okolic Konina. - Biblioteka Monitoringu Srodowiska, Poznan. 2001. - $75 \mathrm{p}$.

Song $W$., Wilbert N. Bentische Ciliaten des Süsswassers. // Praktikum der Protozoologie Eds R. Röttger. Gustav Fischer Verlag, Stuttgart, 1995. - P. 156-168.

Strayer D. L., Lutz C., Malcom H. M., Munger K., Shaw W. H. Invertebrate communities associates with a native (Vallisneria americana) and an alien (Trapa natans) macrophyte in a large river // Freshwater Biology. - 2003. - 48. - P. 1938-1949.

Wetzel R. G. Periphyton of freshwater ecosystems. - Junk : The Hague, 1983. - 346 p.

Wetzel R. G. Limnology: Lake and River Ecosystem, part 19: Land -water interface: attached microorganisms, litoral algae, and zooplankton. - San Diego : Academic Press, 2001. - 1006 p.

Wickham S., Nagle A. S., Hillebrand H. Control of epibenthic ciliate communities by grazers and nutrients // Aquatic Microbial Ecology. - 2004. - 35. - P. 153-162.

Wilbert N. Eine verbesserte Technik der Protargolimprägnation für Ciliaten // Mikrokosmos. - 1975. 64. - P. 171-179. 